\title{
A IMPORTÂNCIA DA VALIDAÇÃO FARMACÊUTICA DA PRESCRIÇÃO MÉDICA E O IMPACTO NA SEGURANÇA DO PACIENTE EM UMA UNIDADE HOSPITALAR
}

\author{
M.M.H. SANTOS ${ }^{1}$, G.R de ALMEIDA ${ }^{1}$, N.M.S. VICENTE ${ }^{2}$ e E.M. SILVA ${ }^{1}$ \\ ${ }^{1}$ Faculdade Pernambucana de Saúde \\ ${ }^{2}$ Real Hospital Português de Beneficência em Pernambuco \\ E-mail para contato: monica-henrique2011@live.com
}

RESUMO: Atualmente, esforços para garantira segurança do paciente nas unidades hospitalares são amplamente discutidos entre os profissionais de saúde. Dentre umas das questões debatidas são os erros na prescrição, já que os mesmos podem levar danos ao paciente e, quando evitados, asseguram uma terapia de melhor qualidade e menor tempo de internamento. Com isso, uma das formas de evitá-los é realizando a validação farmacêutica. O objetivo do trabalho é ressaltar a importância da validação das prescrições medicamentosas, tendo como base os indicadores de validação farmacêutica, no periodo de junho a dezembro de 2016. Estudo retrospectivo, realizado em um hospital de alta complexidade na cidade do Recife. A coleta foi feita de acordo com os indicadores previamente estabelecidos pela instituição do estudo. Os achados corroboram com os poucos estudos nacionais acerca deste tema. Foram quatro indicadores analisados: interação medicamentosa, diluente, incompatibilidade com sonda e duplicidade terapêutica. Quanto aos resultados do indicador de interação, não houve alteração importante. Em relação à diluição, o percentual de erros diminuiu até $15 \%$ em relação ao primeiro mês do serviço. No quesito incompatibilidade no preparo de medicamentos com sonda, no primeiro mês de análise a cada 3 prescrições, 1 possuía erro, e, no último mês, em cada 7 prescrições, 1 tinha erro. O ponto de menor prevalência foi a duplicidade terapêutica com 5\% de participação nos erros. Nos dados estudados nota-se diminuição no número de erros, com exceção da interação medicamentosa. Logo, a validação é fundamental para redução de erros, entretanto, é necessária capacitação, estimulando a educação continuada, e melhorias da equipe farmacêutica em número para, então, obter redução em todos os pontos cabiveis.

Palavras chave: Erros de Medicação. Segurança do Paciente. Indicadores de Serviços. Serviço de Farmácia Hospitalar.

ABSTRACT: Currently, efforts to ensure the patient safety in the hospital are frequently discussed among health professionals. This prescription errors can results in harm to patients, and, when avoided bring a better therapy and reduction of treatment time. The pharmaceutical validation is one of the ways to avoid prescriptions errors. The purpose of this study is to emphasize the importance of pharmaceutical validation of medical prescription, based on the indicators of a hospital of a high complexity, during a period from June to December 2016. Is a retrospective study, performed in a hospital of a high complexity in the city of Recife. The data was extracted from the institution's indicators were the study was done. The results agree with the few national studies on this topic. Were four points analyzed: drug interaction, diluent, incompatibility of the drug with the feeding 
tube, and medication duplicity. About the drug interaction there was no significant change. When talking about the diluent the percentage of errors decreased by $15 \%$ in relation to the first month of the study. At point drug incompatibility with the feeding tube, in the first month, every 3 prescriptions, 1 contained error, and, in the last month, every 7 prescriptions, only 1 contained error. The point that least appears is medication duplicity, with 5\% participation in errors. In the beginning, every 8 prescriptions, 1 contained an error, and, later, every 35 prescriptions, 1 contained error. There is a decrease in the number of errors, except for drug interaction. The pharmaceutical valuation is key to reducing errors, however, training is needed, stimulating continuing education and improvement in the number of pharmaceuticals professionals, to obtain reduction in all the relevant points.

Keywords: Medication Errors. Patient Safety. Indicators of Services. Hospital Pharmacy Service.

\section{INTRODUÇÃO}

Sabendo da importância dos medicamentos na assistência à saúde, o profissional médico no ato da prescrição deve reunir conhecimentos clínicos e farmacológicos para garantir o sucesso da terapia e alívio dos desconfortos dos pacientes. De acordo com relatos de erros de prescrição e administração dos medicamentos, esses foram ocasionados pela falta de atenção e conhecimento farmacológico (OLIBONI. 2009). Nesse contexto, insere-se o erro de medicação que é qualquer evento evitável que possa levar ao uso inadequado de medicamentos podendo ou não provocar danos. Além disso, pode ocorrer em qualquer momento do processo de medicação executadas por uma equipe multidisciplinar (MIASSOL, 2006).

Entre os erros de medicação, encontra-se o erro de prescrição que não corrigidos, resulta em danos aos pacientes. A prescrição deve ser apropriada, com doses adequadas em intervalos definidos nos parâmetros internos, durante o tempo indicado de tratamento e garantir que os medicamentos sejam seguros, eficazes e com qualidade. Prescrições de medicamentos difíceis de interpretar, com ausência de padrões na nomenclatura, uso de abreviaturas e presença de rasuras são fatores que contribuirão para ocorrência de erros. Para evita-los é importante ações de alertas dos erros mais frequentes e os danos que podem causar ao paciente, estabelecendo, assim, uma cultura de comunicação interdisciplinar mais coesa com educação continuada. (CASSANI, 2010).

Em uma validação farmacêutica, os principais pontos, recomendados por instituições acreditadoras de hospitais, a serem observados na análise das prescrições são as interações medicamentosas, incompatibilidade de administração de medicamentos via sonda, erros de diluição, duplicidade terapêutica e alergias. (CBA/JCI 2014). Nesse processo a prescrição médica se configura como mais uma barreira para garantir segurança do paciente no âmbito hospitalar, já que a maioria dos problemas relacionados aos medicamentos seriam evitados. Portanto, o objetivo desse estudo é analisar os indicadores, gerados pela farmácia clinica, das validações farmacêuticas nas prescrições médicas de um hospital de alta complexidade com ênfase na atuação farmacêutica e sua importância na garantia da segurança do paciente.

\section{MATERIAIS E MÉTODOS}

Trata-se de um estudo observacional, retrospectivo, quantitativo, realizado junto à Coordenação da Assistência Farmacêutica (AF) do hospital. Os dados foram coletados através de indicadores de erros de prescrição da Farmácia Clínica no período de junho a dezembro de 2016. O estudo foi conduzido na visão sistêmica do erro, voltado para 
processos e não para pessoas e objetivou analisar a prevalência de erros clinicamente significativos em prescrição, comparando sua ocorrência durante os meses de atividade do farmacêutico clínico como forma de avaliar o impacto desse serviço para a segurança do paciente. As prescrições foram analisadas de acordo com um Procedimento Operacional Padrão (POP) elaborado pela instituição e os principais itens pontuados e analisados por esse trabalho, são: interação medicamentosa, erro de diluente, incompatibilidade com sonda e duplicidade.

\section{RESULTADOS E DISCUSSÃO}

Obteve-se um total de 37.600 prescrições validadas durante o período do estudo de sete meses. Essas prescrições foram analisadas, inicialmente provenientes 165 leitos de UTIs e posteriormente, foi incluído mais 280 de leitos das clínicas médicas e cirúrgicas. A escolha inicial de se validar os leitos das UTIs, reporta-se ao fato do número insuficiente de profissionais farmacêuticos, iniciando, assim, a atividade por pacientes mais críticos. A partir do mês de setembro, com a contratação de mais três profissionais para esta atividade, o número de prescrições validadas aumentou.

De acordo com os dados encontrados no estudo, conforme mostra a figura $1,45 \%$ dos erros identificados se referem a interações medicamentosas, nas quais foram consideradas apenas as interações moderadas e severas. Durante o processo da validação, o farmacêutico, ao identificar este possível erro de prescrição, deve intervir junto ao prescritor, para a correção devida.

Figura 1 - Participação de cada erro no total de erros notificados.

Total de Erros de Prescrição

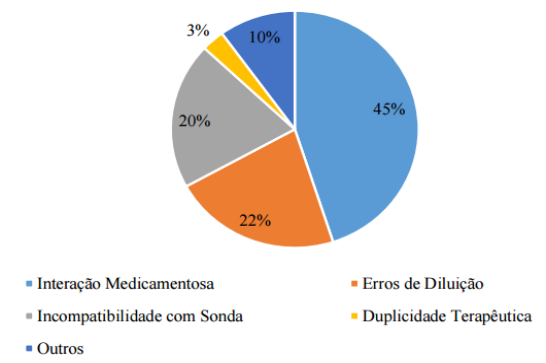

Conforme mostra a figura 1 , os erros de diluição apontam como a segunda causa de erros no estudo, responsável por $22,79 \%$ dos erros encontrados. De acordo com a conduta interna do hospital, o farmacêutico deve sinalizar na validação e entrar em contato com a equipe médica para avisar dos riscos e informar o ajuste indicado. No mês de dezembro, último mês do estudo, a cada cinco prescrições, uma apresenta um erro de diluente. Diante disso, é possível concluir que por meio do serviço de validação prestado pelo profissional farmacêutico, foi possível promover uma diminuição na quantidade de erros neste quesito diluição.

Quanto ao critério incompatibilidade dos medicamentos via sonda, conforme mostra a figura 1 , o item representou $20 \%$ do total de erros no período do estudo. Tais erros representam formas farmacêuticas off labbel que podem ou não serem compatíveis com a estabilidade e farmacocinética do medicamento em uso no momento de sua administração, além de causar obstrução na sonda, trazendo outros danos ao paciente. Além da intervenção do farmacêutico junto ao corpo de enfermagem, esses dados levaram a realização de treinamentos pela equipe de farmacêuticos clínicos ao corpo de enfermagem, a partir de janeiro de 2017. 
O critério duplicidade terapêutica, tem a menor participação no total de erros de prescrição, apresentando $3 \%$ de participação no total dos dados no período do estudo, conforme mostra a figura 1. Configura-se como duplicidade terapêutica quando há prescrito para o mesmo paciente duas drogas de mesmo princípio ativo, em mesma dose e mesma frequência, porém os prescritores usam nomes comerciais diferentes.

Dentre os dados apresentados, o único ponto que não apresentou melhorias depois da implantação do serviço, foi o ponto de interação medicamentosa. Durante a validação farmacêutica, com auxílio de sistemas informatizados e a literatura farmacológica, o profissional deve analisar cada item da prescrição para garantir que não haja uma interação significativa que venha abalar a segurança do paciente no ambiente hospitalar.

\section{CONCLUSÃO}

A prescrição se configura como um instrumento importante de comunicação do médico com a equipe de saúde e paciente dentro do hospital. A atuação do farmacêutico clínico junto à equipe multidisciplinar é fundamental para identificação e prevenção dos erros relacionados aos medicamentos, se configurando como mais uma barreira para evitar danos ao paciente. A validação da prescrição médica funciona como uma barreira para minimizar os erros de medicação. As intervenções farmacêuticas realizadas estão focadas em evitar erros antes que eles aconteçam e possam causar algum dano aos pacientes. Dados apontaram os principais erros de prescrição passíveis de notificação através da validação farmacêutica, onde se identificou que as interações medicamentosas, intencionais ou não, configuram o principal erro. Os resultados obtidos corroboram com poucos achados de estudos nacionais que evidenciam que os erros de prescrição não são raros nos hospitais, com vários setores de pacientes críticos, por exemplo, e devem ser enfrentados pelos profissionais envolvidos na assistência.

\section{REFERÊNCIAS}

CASSANI. S.H.B.; MONZANI. A.A.S; SILVA. A.E.B.C. et al. Identificação e análise dos erros de medicação em seis hospitais brasileiros. Ciência e Enfermagem, 2010, 16(1):85-95.

CBA / JCI - Padrões de acreditação da Joint Comission International para hospitais. $5^{\text {a }}$ Edição [periódico online]. 2014 . Disponível em: <https://www.jcrinc.com/assets/1/14/EBJCIH14B_Sample_Pages.pdf.>

MIASSOL. A.L.; GROULl. C.R.; CASSIANIL. S.H.B.; SILVA. A.E.B.C.; FAKIH. F.T.; Erros de medicação: tipos, fatores causais e providências tomadas em quatro hospitais brasileiros. Rev. esc. enferm. USP vol.40 no.4 .2006.

OLIBONI. L.S.; CAMARGO. A.L.; Validação da prescrição oncológica: o papel do farmacêutico na prevenção de erros de medicação. Rev HCPA 2009;29(2).

Sociedade Brasileira de Farmácia Hospitalar e Serviços de Saúde (SBRAFH). Padrões Mínimos para Farmácia Hospitalar. Organização Conselho Federal de Farmácia Goiânia: [periódico online]. 2007. Disponível em: <http://www.sbrafh.org.br/site/public/temp/4f7baaa6b63d5.pdf> 